\title{
A educação em saúde como agente promotor de qualidade de vida para o idoso
}

\section{Education in health as a life quality promoter for elderly people}

M ônica Cristina de M elo ${ }^{1}$

André Luiz Souza ${ }^{1}$

Edélvio Leonardo Leandro ${ }^{1}$

Herika de Arruda M auricio ${ }^{1}$

Iêdo Donato Silva ${ }^{1}$

Juliana Maria Oriá de Oliveira ${ }^{1}$

Abstract This article presents considerations on the education in health as a life quality promoter in order to reach actions and behavior conditions for the Brazilian elderly community health. From a literature review, it could be noticed that the educational work has as challenge the integration of the huge knowledgein human and biological areasto the common knowledge, improving new policies in the model of the health care system. The education in health reveals to be the link between the desires and expectations of this population for a better life and the projections and policies of the government to offer more efficient health care programs.

Key words Elderly people, Education in health, Quality of life
Resumo Este artigo tece consi derações sobre a educação em saúde como agente promotor da qualidade de vida a fim de atingir ações e condi ções conducentes à saúde do idoso no contexto brasileiro. A partir de uma revisão da literatura na área, verificou-se que o trabalho educacional tem como desafio a integração de conhecimentos dispersos das áreas humanas e biológicas aos saberes populares, pressupondo novas interfaces de atuação no modelo de assistência à saúde. A educação em saúde desponta como um elo entre os desejos e expectativas dessa população por uma vida melhor e as projeções e estimativas dos governantes ao oferecer programas de saúde mais eficientes.

Palavras-chave Idoso, Educação em saúde, Qualidade devida

${ }^{1}$ Curso de especialização

em SaúdeColetiva,

Faculdade M aurício de

Nassau. Rua Guilherme

Pinto 114 , Graças.

52011-210 Recife PE.

monicademelo@ig.com.br 
Introdução

A humanidade está passando por um processo de mudança populacional conhecido por "transição demográfica", em que as diferentes sociedades humanas estão deixando, por condições diferentes ( redução da fecundidade, da mortalidade infantil e também da mortalidade em idades mais avançadas), de ser sociedades em que predominam as populações jovens e maduras para se transformarem em sociedades cada vez mais envelhecidas ${ }^{1-3}$.

A Organização das Nações Unidas (ONU) considera o período de 1975 a 2025 a era do envelhecimento. N os países em desenvolvimento, esse enve Ihecimento populacional foi ainda mais significativo e acelerado, destaca a ONU: enquanto nas nações desenvolvidas, no período de 1970 a 2000, o crescimento observado foi de $54 \%$, nos países em desenvolvimento, atingiu $123 \%$. No Brasil, segundo dados do IBGE, na década de setenta, cerca de $4,95 \%$ da população brasileira era de idosos, percentual quepulou para $8,47 \%$ na década denoventa, havendo a expectativa deal cançar 9,2\% em 2010 . 0 aumento do número deidosos também tem sido acompanhado por um acréscimo significativo nos anos de vida da população brasileira. A esperança de vida, que era em torno de 33,7 anos em 1950/ 1955, passou para 50,99 em 1990, chegou a 66,25 em 1995 edeveráalcançar 77,08 em 2020/20254.

Do ponto de vista biológico, o envelhecimento é descrito como um estágio de degeneração do organismo, quese iniciaria após o período reprodutivo. Essa deterioração, que estaria associada à passagem do tempo, implicaria uma diminuição da capacidade do organismo para sobreviver. Entretanto, o problema começa quando se tenta marcar o início desse processo, ou medir o grau desse enveIhecimento/degeneração. Por maisincrível quepossa parecer, o critério mais comumente utilizado para a definição do envelhecimento - o cronológico (aidade) - é apontado como falho e arbitrário. Isso porque 0 envelhecimento seria vivenciado de forma heterogênea pela população. Pessoas da mesma idade cronológica poderiam estar em estágios completamente distintos de envelhecimento. Além disso, o próprio organismo de um indivíduo "enveIheceria" demaneira diferente entreos seus tecidos, ossos, órgãos, nervos e células. D esse modo, o envelhecimento não parece ser definido pela idade de uma pessoa, mas pelos efeitos que essa idade teria causado a seu organismo ${ }^{5}$. Nessa perspectiva, a veIhice é percebida como fenômeno natural e social quese desenrola sobreo ser humano, único, indivisível, que, na sua totalidade existencial, defronta-se com problemas e limitações de ordem biológica, econômica e sociocultural, que singularizam seu processo de envel hecimento. Dessemodo, somente uma descrição analítica dos diferentes aspectos da velhicenão éconsiderada suficiente para explicá-la, visto que cada um desses aspectos interage com todos os outros e é por eles afetado $0^{4,6}$.

0 envelhecimento populacional constitui um dos maiores desafios para a saúde pública contemporânea, especialmente em países em desenvolvimento, onde este fenômeno ocorre em ambiente de pobreza egrande desigualdade social ${ }^{7}$. Com o aumento da expectativa de vida dos indivíduos, modifica-se, também, o seu perfil de saúde; em vez de processos agudos ou de óbito, tornam-se predominantes as doenças crônicas e suas complicações, como a perda da sua autonomia e independência funcional, que, além de demandarem maiores custos para os serviços de saúde, exigem um reordenamento das suas ações prioritárias. M esmo sendo esse o cenário atual, observa-se, no geral, que os serviços de saúde não estão preparados para responder às necessidades múltiplas dos ido$\mathrm{sos}^{2}$. A maioria das doenças crônicas que acomete o indivíduo idoso tem, na própria idade, seu principal fator de risco ${ }^{8}$.

Não obstante, conforme Lima-Costa ${ }^{3}$, os idosos são grandes usuários de serviços de saúde, sendo este fenômeno consequência da maior prevalência de doenças e deincapacidades nessa população. Em países desenvolvidos, o uso de serviços de saúde entre idosos é cerca de três a quatro vezes maior que o seu tamanho proporcional na população. No Brasil, o custo proporcional das internações hospitalares públicas entre idosos é três vezes maior do que o tamanho proporcional desta no conjunto da população brasileira. Apesar de a atenção à saúde ser um direito universal no Brasil, o acesso no uso de serviços pela população idosa é fortemente influenciado pela situação socioeconômica do idoso e/ou da sua família. Os idosos com menor renda domiciliar mensal apresentam piores condições de saúde em comparação com aqueles com melhor situação socioeconômica, mas visitam médicos com menos frequência.

M esmo com a extensão da atenção à saúde, ocorrida a partir dos anos oitenta, aborda-se o idoso, na maioria das vezes, de modo limitado às enfermidades crônicas e em consultas individuais esporádicas, sem continuidade, e desconsiderando o impacto desse quadro na qualidade de vida. A precária assistência ao idoso pode ser constatada pela elevada proporção de óbitos por causas mal definidas (que chega a 65\%) e à subnotificação de problemas considerados esperados ou normais para a idade e não passíveis de intervenção. 
As enfermidades crônicas apresentam-se de modo simultâneo e múltiplo e seu caráter insidioso e, muitas vezes, subclínico, dificulta o diagnóstico ea aderência ao tratamento. Por tais razões, 0 acompanhamento do idoso requer o autoconhecimento das enfermidades, complicações e indicaçõesterapêuticas, bem como a motivação e educação contínua e de modo compartilhado ${ }^{9}$.

M uitas vezes, na vel hice, os problemas de saúde causados por patologias múltiplas são agravados pela solidão e pobreza ${ }^{10}$. A inatividade e a falta de perspectivas na aposentadoria podem levar a um sentimento dedepressão que consequentemente compromete a saúde do indivíduo ${ }^{11}$. Especificamente sob a presença de suportes sociais, é esperado que pessoas idosas sintam-se amadas, seguras para lidar com problemas de saúde e tenham alta auto-estima, reduzindo os efeitos negativos do estressena saúde mental, tendo uma influência positiva no bem-estar psicológico ${ }^{12}$.

No Estatuto do Idoso, é assegurada a atenção integral à saúde, por intermédio do Sistema Ú nico deSaúde (SUS), garantindo-Iheo acesso universal e igualitário, em conjunto articulado e contínuo das ações e serviços, para a prevenção, promoção, proteção erecuperação da saúde, incluindo a atenção especial às doenças que afetam preferencialmente os idosos ${ }^{13}$. 0 acesso a serviços de saúde de qualidade é um elemento central para a qualidade de vida relacionada à saúde do idoso ${ }^{3}$.

Para a Organização M undial de Saúde (OMS), a qualidade de vida é definida como "percepção do indivíduo sobre a sua posição na vida, no contexto da cultura e dos sistemas de valores nos quais ele vive, e em relação a seus objetivos, expectativas, padrões e preocupações". N essa definição, incluem-se seis domínios principais: saúde física, estado psicológico, níveis deindependência, relacionamento social, características ambientais e padrão espiritual. N esse contexto, serviços que contribuem para promoção de uma qualidade de vida voltada para a sua população idosa tornam-se um desafio, tendo em vista que devem considerar uma valoração subjetiva que o próprio idoso faz de diferentes aspectos de sua vida em relação ao seu estado de saúde $e^{14,15}$.

\section{Revisão crítica}

Q ualidade de vida éuma expressão que vem setornando corriqueira no dia a dia das pessoas, mas queserevestedegrande complexidade, dada a subjetividade que representa para cada pessoa ou grupo social ${ }^{16}$. Por outro lado, em um nível abstrato, al- guns autorestêm considerado queexisteum conceito "universal cultural" de qualidade de vida, isto é, independente da nação, cultura ou época, é importantequeas pessoas se sintam bem psicologicamente, possuam boas condições físicas esintam-se socialmente integradas efuncionalmentecompetentes ${ }^{17}$.

0 termo qualidade de vida tem movido o interesse das Nações Unidas desde a década de cinquenta. Uma boa parte das investigações, inicialmente, associava a qualidade de vida a um conceito quantitativo, relativo a recursos materiais disponíveis para determinado indivíduo ou sociedade. Atualmente, existe uma abordagem mais ampla e integradora que leva em consideração as necessidades básicas do ser humano em direção ao sentimento de bem-estar subjetivo e social ${ }^{18}$. As questões de qualidade de vida têm avançado em níveis consideráveis, sob o enfoque dos determinantes sociais de condição de vida e desenvolvimento ${ }^{19}$. Uma qualidade de vida boa ou excelenteé aquela que oferece um mínimo de condições para que os indivíduos possam desenvolver o máximo de suas potencialidades, sejam estas viver, sentir ou amar, trabalhar, produzindo bens e serviços, fazendo ciências ou artes ${ }^{20}$.

Rogerson em Rocha et al. ${ }^{19}$ desenvolve dois modelos conceituais sobre qualidade de vida: um relacionado à saúde e sua recuperação e outro relacionado às questões ambientais. 0 primeiro enfoqueenvolvea questão de recuperação da saúdeapós um evento de doença ou agravo. 0 segundo é 0 reflexo da realidade multifacetada de determinado grupo social, com ênfase nas distorções desse meio para enfrentamento das iniquidades. 0 modelo incorpora aspectos comportamentais e perceptivos da comunidade acerca da qualidade de vida.

No campo da saúde, a relação entre saúde e qualidade de vida existe desde 0 nascimento da medicina social, nos séculos XVIII eXIX. A expressão qualidade de vida em saúde utilizada na área médica dentro do referencial da clínica possui, no entanto, uma visão medicalizada, com indicadores fundamentados em uma lógica de custo-benefício. Redimensionada pelo pensamento sanitarista, a concepção atual considera como determinantes de saúde o estilo de vida; avanços da biologia humana; ambiente físico e social; e serviços de saúde ${ }^{21}$.

Devido a essa ausência de definição consensual sobre qualidade de vida, torna-se difícil mensurála. Para medir qualidade de vida, um ponto sugere indagação: de quem éa melhor percepção, daqueles que a vivem ou daqueles quea observam? Possivel mente ambos, desde que os aspectos humanos e os do espaço urbano se fundam em um conceito agregado de desenvolvimento humano e sustentá- 
$v^{2}{ }^{19}$. Existem duas formas de mensuração: através de instrumentos genéricos e instrumentos específicos. Os genéricos abordam o perfil de saúde ou não, procuram englobar todos os aspectos importantes relacionados à saúde e refletem o impacto de uma doença sobre o indivíduo. Os específicos avaliam de maneira individual eespecífica determinados aspectos da qualidade de vida22. A maioria dos instrumentos de avaliação foi desenvolvida nos Estados Unidos, com um crescente interesse em traduzi-los para aplicação em outras culturas. A aplicação transcultural através da tradução é um tema controverso. A busca de um instrumento que avaliasse a qualidade de vida dentro de uma perspectiva de saúde internacional fez com que a Organização Mundial de Saúde (OMS) desenvolvesse o WHOQOL-100 e OWHOQOL - Bref. O primeiro é um instrumento de avaliação composto por cem itens e que já possui uma versão em português. 0 segundo é uma versão abreviada com 26 questões extraídas do anterior ${ }^{17,21}$. Outro exemplo importante de análise de qualidade de vida é o Índice de Desenvolvimento Humano (IDH), que tem como sistemática a visão total do espaço considerado. 0 IDH utiliza três componentes de desenvolvimento humano: longevidade, grau de conhecimento erenda ou PIB per capita, por entender queestes indicadores refletem o todo da realidade em análise ${ }^{19}$.

Osinstrumentos utilizados para análise da qualidade de vida, de um modo geral, não se adaptam aos idosos, seja por possuírem uma abordagem unidimensional ou por levarem em conta aspectos característicos que osidosos não consideram como promotores de qualidade de vida. A pesquisa realizada no município de Botucatu, São Paulo, comprovou que entre os idosos existem grupos com diferentes perfis e cada grupo faz uma diferente interpretação sobre qualidade de vida ${ }^{23}$. Avaliar a qualidade de vida do idoso implica a adoção de múltiplos critérios de natureza biológica, psicológica e socioestrutural, pois vários elementos são apontados como determinantes ou indicadores de bem-estar na velhice: longevidade, saúde biológica, saúde mental, satisfação, controle cognitivo, competência social, produtividade, atividade, eficácia cognitiva, status social, renda, continuidade de papéis familiares, ocupacionais e continuidade de relações informais com amigos ${ }^{20}$. O EASY careé um sistema de avaliação de idosos desenvolvido com o objetivo de caracterizar a qualidade de vida e bem-estar deste grupo. Trata-se de um sistema de avaliação multidimensional, mas apresenta limitações psicométricas, exigindo a revisão de alguns iten ${ }^{24}$. Reveste-se de grande importância científica e social analisar as condições de vida na terceira idade, por permitir a implementação de alternativas válidas de intervenção, tanto em programas gerontogeriátricos, quanto em políticas sociais gerais, no intuito de promover o bem-estar das pessoas maduras ${ }^{20}$. M as a interpretação de indicadores deve ser apenas entendida como reflexo minoritário da realidade complexa do dia a dia ${ }^{19}$.

As estratégias de promoção de saúde devem voltar-se para estilos de vida e condições sociais, econômicas e ambientais que determinam a saúde e, de forma mais ampla, a qualidade de vida ${ }^{18}$. A promoção de saúde representa uma forma promissora para enfrentar os múltiplos problemas de saúde que afetam as populações humanas, propondo a articulação dos saberes técnico e popular, e a mobilização de recursos institucionais e comunitários, públicos e privados, para seu enfrentamento e resolução 25 .

A Carta de Ottawa foi um dos documentosfundadores do termo promoção de saúde, definindoa como o processo de capacitação da comunidade para atuar na melhoria de sua qualidade de vida e saúde, incluindo uma maior participação no controle desse processo ${ }^{25}$. Modernamente, a promoção de saúde caracteriza-se pelo entendimento que a saúde é produto de um amplo espectro de fatores relacionados com a qualidade de vida, incluindo um padrão adequado de alimentação e nutrição, e de habitação e saneamento; boas condições de trabalho; oportunidades deeducação ao longo da vida; ambiente físico limpo, apoio social para famílias e indivíduos; estilo de vida responsável; e um espectro adequado de cuidados de saúde ${ }^{25}$.

Os mecanismos operacionais concretos para a implementação da estratégia da promoção da saúde e da qualidade de vida são políticas saudáveis, governabilidade, gestão social integrada, intersetorialidade, estratégias de municípios saudáveis e desenvolvimento local, com ênfase particular no contexto do nível local ${ }^{25}$. A existência de programas integrados é uma boa estratégia para atacar 0 ciclo de pobreza-doença-sofrimento ${ }^{26}$. Isto implica responsabilidades para os profissionais de saúde, para os movimentos sociais e organizações populares, políticos e autoridades públicas².

A promoção de saúde e a profilaxia primária e secundária de doenças, inclusive após os 65 anos, são as alternativas que apresentam o melhor custo-benefício para que se alcance a compressão da morbidade. Sua importância deveser enfatizada nos cursos de graduação e de educação continuada da área da saúde ${ }^{27}$. A realização de estudos que visem pesquisar concepções sobre o envel hecimento ajudaria na construção de conteúdos para formação e capacitação de pessoal, instrumento para gestão e 
como apoio na tomada de decisões, por meio da avaliação para gestão ${ }^{28}$. A prestação deserviços para idosos deve ser precedida por um diagnóstico epidemiológico que possibilite um planejamento adequado à realidade socioeconômica das diversas regiões brasileiras, sendo que o enfoque sistemático em relação aos serviços para os idosos é, por definição, multidisciplinar emultisetorial ${ }^{29}$.

Segundo Silvestre e Costa Neto ${ }^{30}$, o trabal ho na atenção básica sob a Estratégia de Saúde da Família é uma adequada abordagem da pessoa idosa. Entretanto, as equipes de saúde da família não podem perder de vista que o estresse de agravos físicos, emocionais e sociais, com o passar do tempo e, consequentemente, com o aumento da idade, representa uma efetiva e progressiva ameaça para saúde da pessoa idosa. Frente a tal realidade, o profissional de saúde enfrenta o desafio de traçar limites entre o que se pode considerar como envel hecimento normal com suas limitações fisiológicas gradativas e as características patológicas que podem instalar-se durante esse processo. Diversos fenômenos que eram tidos como normais com o avançar da idade hoje são considerados como decorrentes da instalação de processos patológicos e devem ser precocementeidentificadose trabalhados de forma participativa e efetiva.

0 envel hecimento da população éuma questão que extrapola a esfera familiar e, portanto, a responsabilidade individual, para alcançar o âmbito público, neste compreendido o Estado, as organizações não-governamentaise os diferentes segmentos sociai $5^{31}$. Além disso, pela diversidade da abrangência e de localização geográfica, as entidades de mobilização comunitária podem se transformar em parceiras tanto para pequenos e específicos programas de educação em saúde, como para grandes campanhas nacionais de comunicação de massas ${ }^{32}$.

Seguindo este modelo, foram concebidos grupos de promoção à saúde como instrumentos a serviço da autonomia e do desenvolvimento contínuo do nível de saúde e condições de vida do idoso. Entre eles, o Grupo de Atenção à Saúde do Idoso (GRASI) em Campinas, o Programa deAtenção à Saúde em João Pessoa, Grupo de Apoio Social oferecido a Idosos da Área Rural em Taquarituba. Ao atuarem no campo comunitário, os grupos abrem possibilidades para as ciências da saúde e do homem, horizontes que vão além do simples objetivo de combater as doenças dos indivíduos. Estes deverão acrescer à sua tarefa a preocupação com a própria identidade da pessoa humana na busca do grau mais elevado possível de saúde física, mental e social para si e para a sociedade em que vivem. Trata-se da aprendizagem cidadã des- tacada por M orin: "A educação deve contribuir para a autoformação da pessoa (ensinar a assumir a condição humana, ensinar a viver) e ensinar como se tornar cidadão" 33 .

M as é preciso fazer a diferenciação entre educação em saúde e promoção em saúde para que não existam distorções conceituais. Entende-sepor educação em saúde quaisquer combinações de experiências de aprendizagem delineadas com vistas a facilitar ações voluntárias conducentes à saúde. Enquanto que promoção em saúde é uma combinação de apoios educacionais e ambientais que visam atingir ações econdições devida conducentes à saúde. Dessa forma, a educação em saúde procura de sencadear mudanças de comportamento individual, enquanto que a promoção em saúde, muito embora inclua sempre a educação em saúde, visa provocar mudanças de comportamento organizacional, capazes de beneficiar a saúde de camadas mais amplas da população, particularmente, porém não exclusivamente, por meio da legislação ${ }^{34}$.

Entende-se por educação em saúde um campo de práticas que se dão no nível das relações sociais normalmente estabelecidas pelos profissionais de saúde entre si, com a instituição e sobretudo com o usuário, no desenvolvimento cotidiano de suas atividades. Não se pode pensar os serviços de saúde sem refletir sobre as relações entre esses atores ou sujeitos, uma vez que qualquer atendimento à saúde envolve, no mínimo, a interação entre duas pessoas $^{35}$. A Organização Mundial de Saúde pontua que os objetivos da educação em saúde são de desenvolver nas pessoas o senso de responsabilidade pela sua própria saúdeepela saúdeda comunidade a qual pertençam e a capacidade de participar da vida comunitária de uma maneira construtiva. Estas assertivas fazem da educação em saúde um dos mais importantes el os entre os desejos e expectativas da população por uma vida melhor e as projeções e estimativas dos governantes ao oferecer programas de saúde mais eficientes ${ }^{32}$.

Em qual quer sistema de saúde, não se pode conceber o planejamento deação sem antes considerar as premissas do planejamento educativo. Se isso ocorrer, a prática subsequente será equivocada e, portanto, ilógica no que diz respeito às necessidades da população-alvo que se pretende alcançar. Na prática, a educação em saúde constitui apenas uma fração das atividades técnicas voltadas para a saúde, prendendo-se especificamente à habilidade de organizar logicamente o componente educativo de programas que se desenvolvem em quatro diferentes ambientes: a escola, o local de trabalho, 0 ambiente clínico em seus diferentes níveis de atuação ea comunidade, compreendida aqui como con- 
tendo populações-alvo que não se encontram normalmente nas três outras dimensões ${ }^{34}$.

Do ponto de vista biopsicológico, a educação tem por objetivo levar o indivíduo a realizar suas possibilidades intrínsecas, com vistas à formação e ao desenvolvimento desua personalidade. Sociologicamente, a educação é um processo que tem por fim conservar e transmitir cultura, atuando como importante instrumento e técnica social. A aspiração do trabalho a ser desenvolvido na área de educação em saúde está voltada para a auto-educação (onde existea participação intencional do educando em procurar influências capazes de lhe modificar o comportamento e submeter-se a elas), pois exprimea tomada deconsciência por parte do indivíduo acerca da importância do processo educativo para a sua formação e desenvolvimento ${ }^{32}$.

0 programa educativo tem como premissa 0 envolvimento de todos os membros do grupo, por meio da integração e da interação dos profissionais com os idosos e seus familiares. A participação familiar ou de uma pessoa próxima do idoso no programa de educação à saúde é fundamental para a compreen são das implicações que o processo de envel hecimento traz na vida diária do indivíduo, bem como de seus desdobramentos no contexto familiar. Se a motivação eo interesse dos idosos e familiares não estiverem presentes, 0 trabaIho estará destinado ao fracasso, mesmo com todos os esforços do educador 6 .

A maioria das instituições de ensino superior brasileiras ainda não está sintonizada com o atual processo de transição demográfica e suas consequências médico-sociais. Há uma escassez de recursos técnicos e humanos para enfrentar a explosão dessegrupo populacional no terceiro milênio ${ }^{31}$. A demanda dos profissionais pode ser dividida em duas direções: a instrumentalização em técnicas didático-pedagógicas e o desenvolvimento do papel profissional. Os profissionais devem ser capacitados para a busca constante das relações sociais quese desenvolvem no dia a dia dos serviços, numa perspectiva crítica de visualizar, com naturalidade, os problemas advindos da convivência humana, em qualquer situação na qual ela ocorra. Muito mais importante do que aprender técnicas é adquirir a postura de educador. As técnicas só devem ser buscadas como recurso auxiliar ao processo educativo, porque o principal "instrumento" da relação educativa é o próprio educador. 0 que se pretende com a educação em saúde é contribuir para o desenvolvimento de profissionais como sujeitos autônomos que constroem essa autonomia com base na imaginação de um futuro qualitativamente melhor ${ }^{35}$.
Outros aspectos a serem considerados nas atividades de ensino aos idosos são os fatores fisiológicos, sociais, econômicos e culturais, como alteração da memória, comprometimento sensorial, escolaridade e deficiência de sistemas de suporteà saúde e da rede social de apoio ${ }^{6}$.

A resistência popular às ações sanitárias é conhecida desde a implantação das primeiras políticas de saúde pública no início do século $X X^{36}$. Esta resistência deve-se ao fato de que a política implementada baseia-se em uma visão tradicional de transmissão deconhecimento, limitando-seà prescrição de comportamentos individuais e organização social adequados à obtenção de boa saúde. $\mathrm{N}$ ão é possível al cançar bons resultados na educação em saúde através de programas que pretendem apenas conscientizar, educar, despertar, sensibilizar a população ou identificar carências. É preciso valorizar os sujeitos naquilo que têm de meIhor, sua história, seu tempo - a sua capacidade de criação. 0 que motiva as pessoas não é apenas a sua carência, mas principalmenteo que setem disponível (desejos, sonhos, projetos). Os esforços para a construção de novos conhecimentos, em vez de se voltarem apenas para a busca incessante deacúmulo de informação que representea verdade, devem se voltar para a melhoria das condições de convivência entre distintos saberes e sabedores, compartilhando saberes e a existência. A produção do conhecimento deve ter como ponto de partida a ação social e as informações que emergem dessa convivência têm um potencial de mobilização social. A penas a tomada de consciência dos problemas não é suficiente para motivar os sujeitos, pois o limite de sua ação é dado pela sustentabilidade oferecida pelos grupos de convívio social. Na convivência, não basta apontar "o que fazer", é preciso estar disposto a construir junto "o como fazer". A educação em saúde deve fortalecer a individualidade a partir da disponibilização de suportes coletivos, bens materiais e bens espirituais compaixão, solidariedadee respeito -, para o exercício de uma individualidade saudável ${ }^{37}$.

As ações coletivas podem ser desenvolvidas como estratégias eficientes para a melhoria da qualidade de vida da população, quando sefundamentam na intersetorialidade, na transdisciplinaridade e no desenvolvimento de autonomia dos sujeitos, já que permitem não somente a discussão dos problemas que afetam a comunidade, como também possibilitam a construção coletiva de estratégias de interven ção ${ }^{38}$. A cidadania representa a ligação entre qualidade de vida e políticas públicas, na medida em que a consciência de cidadania significa 0 fortalecimento do poder de participação ${ }^{16}$. 


\section{Considerações finais}

D efender a presença do idoso na família e na sociedade de forma participativa e construtiva é fundamental para que este ser humano tenha respeitado seu direito à saúde com qualidade de vida.

Uma comunidade saudável seria aquela capaz deidentificar eentender os determinantes e condicionantes das desigualdades, construindo meios para superá-los de modo a promover a integração dos idosos com toda a sociedade.

Diante da necessidade de programas de saúde mais eficientes para a terceira idade, o meio de superação encontra-se na educação em saúde.

\section{Colaboradores}

MC M elo trabalhou na concepção e orientação. AL Souza, EL Leandro e ID Silva trabalharam na concepção epesquisa bibliográfica. H A M auricio e JM O Oliveira trabalharam na concepção, pesquisa bibliográfica e redação final.

\section{Referências}

1. Jordão Netto A. A gerontologia básica. São Paulo: Lemos Editorial; 1997.

2. Fernandes MGM, Gonçalves MCR, Costa GMC, Silva SM M . O Programa de Atenção à Saúde do Idoso em João Pessoa - PB: realidade e possibilidades. Conc. João Pessoa 2002; 5(7):146-154.

3. Lima-Costa M F. Epidemiologia do envelhecimento do Brasil. In: Rouquayrol MZ, Almeida Filho N. Epidemiologia \& Saúde. 6a ed. Rio de Janeiro: MEDSI; 2003. p.499-513.

4. Siqueira RL, Botelho MIV, Coelho FM G. A velhice: algumas considerações teóricas e conceituais. Cien Saude Colet 2002; 7(4):899-906.

5. Groisman D. A velhice, entre o normal e o patológico. História, Ciências, Saúde - M anguinhos 2002; 9(1):61-78.

6. Diogo MJD, Ceolim M F, Cintra FA. Implantação do Grupo de Atenção à Saúde do Idoso (GRASI) no Hospital das Clínicas da Universidade Estadual de Campinas (SP): relato de experiências. Rev Latino-am enfermagem 2000; 8(5):85-90.

7. World $\mathrm{H}$ ealth Organization. Population aging: a public health challenge. Geneva: World Health Organization Press Office; 1998.

8. Gordilho A, N ascimento JS, Silvestre J, Ramos LR, Freire M PA, Espindola N, Maia R, Veras R , Karsch $U$. Desafios a serem enfrentados no terceiro milênio pelo setor de saúde na atuação integral ao idoso. Bahia Análise \& Dados 2001; 10(4):138-153.

9. Garcia MAA, Yagi GH, Souza CS, O doni APC, Frigério $\mathrm{RM}, \mathrm{M}$ erlin SS. Atenção à saúde em grupos sob perspectiva dos idosos. Rev Latino-am enfermagem 2006; 14(2):175-182.

10. Veras RP, Ramos LR, Kalache A. Crescimento da população idosa no Brasil: transformações e consequências na sociedade. Rev. Saude Publica 1987; 2(3):225233. 
11. França L. Preparação para a aposentadoria: desafios a enfrentar. In: Veras $R$, organizador. Terceira idade: alternativas para uma sociedade em transição. Rio de Janeiro: Relume Dumará/UNATI; 1999. p. 11-34.

12. Ramos M P. Apoio social e saúde entre idosos. Sociologias 2002; (7):156-175.

13. Brasil. M inistério da Saúde. Estatuto do Idoso. 2ª ed. Revista. Brasília: Ministério da Saúde; 2006.

14. World Health Organization. Quality of life assessment: an annotated bibliography. Geneva: World Health Organization; 1994.

15. Guiteras AF, Bayés R. Desarrollo de un instrumento para la medida de la calidad de vida en enfermedades crónicas. In: Forns M, Anguera MT, compiladores. Aportaciones recientes a la evaluación psicológica. Barcelona: Universitas; 1993. p. 175-195.

16. Queiroz CMB, Sá ENC, Assis MA. Qualidade de vida e políticas públicas no município de Feira de Santana. Cien Saude Colet 2004; 9(2):411-421.

17. Fleck MPA, Leal OF, Louzada S, Xavier M, Chachamovick E, Vieira G, Santos L, Pinzon V. Desenvolvimento da versão em português do instrumento de avaliação de qualidade de vida da OMS (WHOQOL100). Rev. Bras. Psiquiatr. 1999; 21(1):19-28.

18. Souza RA, Carvalho AM. Programa de Saúde da Família e qualidade de vida: um olhar da Psicologia. Estud. Psicol. (Natal) 2003; 8(3):515-523.

19. Rocha $A D, O$ kabe I, M artins MEA, M achado PHB M ello TC. Qualidade de vida, ponto de partida ou resultado final? Cien Saude Colet 2000; 5(1):63-81.

20. Santos SR, Santos IBC, Fernandes M GM , Henriques MERM. Qualidade de vida do idoso na comunidade: aplicação da Escala de Flanagan. Rev Latino-am enfermagem 2002; 10(6):757-64.

21. M inayo M CS, Hartz ZMA, Buss PM. Qualidade de vida e saúde: um debate necessário. Cien Saude Colet 2000; 5(1):7-18.

22. Dantas RAS, Sawada NO, Malerbo MB. Pesquisas sobre qualidade de vida: revisão da produção científica das Universidades Públicas do Estado de São Paulo. Rev Latino-am enfermagem 2003; 11(4):532-538.

23. Vecchia RD, Ruiz T, Bochi SCM, Corrente JE. Qualidade de vida na terceira idade: um conceito subjetivo. Rev. Bras. Epidemiol. 2005; 8(3):246-252.

24. Souza L, Galante H, Figueiredo D. Qualidade de vida e bem-estar dos idosos: um estudo exploratório na população portuguesa. Rev. Saude Publica 2003; 37(3):364-371.

25. Buss PM. Promoção de saúde e qualidade de vida. Cien Saude Colet 2000; 5(1):163-177.
26. Blaxter M. Health services as a defense against poverty in industrialized societies. Social Science and M edicine 1983; 17:1139-1148.

27. Chaimowicz F. A saúde dos idosos brasileiros às vésperas do século XXI: problemas, projeções e alternativas. Rev. Saude Publica 1997; 31(2):184-200.

28. Bezerra AFB, Espírito Santo ACG, Batista Filho M . Concepções e práticas do agente comunitário na Atenção à Saúde do Idoso. Rev. Saude Publica 2005; 39(5):809-815.

29. Guimarães RM. Proteção e saúde do idoso. Rev. Saude Publica 1987; 21(3):274-275.

30. Silvestre JA, Costa N eto M M. Abordagem do idoso em Programas de Saúde da Família. Cad Saude Publica 2003; 19(3):839-847.

31. Brasil. Portaria do Gabinete do Ministro do Estado da Saúde de no 1.395, de 10 de dezembro de 1999. Aprova a Política Nacional de Saúde do Idoso e dá outras providências. Diário Oficial da União 1999; 10 dez.

32. Levy SN, Silva JJC, Cardoso IFR, Werberich PM, Moreira LLS, M ontiani H, Carneiro RM. Educação em saúde: histórico, conceitos e propostas. Brasília: M inistério da Saúde; 1997.

33. Santos LM, Da Ros MA, Crepaldi MA, Ramos LR. Grupos de promoção à saúde no desenvolvimento da autonomia, condições de vida e saúde. Rev. Saude Publica 2006; 40(2):346-352.

34. Candeias NM F. Conceitos de educação e de promoção em saúde: mudanças individuais e mudanças organizacionais. Rev. Saude Publica 1997; 31(2):209-213.

35. L'abbate S. Educação em saúde: uma nova abordagem. Cad Saude Publica 1994; 10(4):481-490.

36. Costa NR. Desenvolvimento das políticas de saúde pública. In: Costa NR. Lutas urbanas e controle sanitário: origens das políticas de saúde no Brasil. $2^{a}$ ed. Petrópolis: Vozes; 1986. p. 19-31.

37. Oliveira RM. A construção do conhecimento nas práticas de educação em saúde: repensando a relação entre profissionais dos serviços e a população. Perspect. Ciênc. Inf. 2003; 8(N. especial):22-45.

38. Wimmer GF, Figueiredo GO. Ação coletiva para qualidade de vida: autonomia, transdisciplinaridade e intersetorialidade. Cien Saude Colet 2006; 11(1):145-154.

Artigo apresentado em 27/12/2006 Aprovado em 13/12/2007 